(2) Open Access Full Text Article

ORIGINAL RESEARCH

\title{
Exosomes-Coated miR-34a Displays Potent Antitumor Activity in Pancreatic Cancer Both in vitro and in vivo
}

This article was published in the following Dove Press journal:

Drug Design, Development and Therapy

\author{
Ling Zuo (D) \\ Hongyu Tao $\mathbb{D D}^{2}$ \\ Huanli $\mathrm{Xu}^{2}$ \\ Cong $\mathrm{Li}^{2}$ \\ Gan Qiao (1D ${ }^{1,3}$ \\ Mingyue Guo (D) ${ }^{\prime}$ \\ Shousong Cao' \\ Minghua Liu (D) \\ Xiukun Lin' \\ 'Department of Pharmacology, School of \\ Pharmacy, Southwest Medical University, \\ Luzhou, Sichuan 646000, People's \\ Republic of China; ${ }^{2}$ Department of \\ Pharmacology, School of Basic Medicine, \\ Capital Medical University, Beijing \\ I00069, People's Republic of China; \\ ${ }^{3}$ Central Nervous System Drug Key \\ Laboratory of Sichuan Province, Luzhou, \\ Sichuan 646000, People's Republic of \\ China
}

Correspondence: Xiukun Lin; Minghua Liu Tel +86830 316 2291;

$+868303193872$

Email xiukunLin@I26.com; liumhvip@|63. com
Purpose: MiR-34a, which acts as an important tumor suppressor gene, plays an important role in pancreatic cancer. However, the therapeutic application of miR-34a is limited by the lack of an effective delivery system. In the present study, we synthesize exosomes-coated miR-34a (exomiR-34a), and the anticancer effect of exomiR-34a was evaluated in pancreatic cancer.

Materials and Methods: An ultrasound approach was used to synthesize exomiR-34a, and its transfection efficiency was examined by confocal microscopy and flow cytometry. The level of miR-34a and its targeted gene Bcl-2 was detected by real-time quantitative PCR (qRT-PCR). MTT analysis was performed to determine the effect of exomiR-34a on the growth of pancreatic cancer cells. Annexin-V/PI double staining and Western blot analysis were carried out to determine the apoptosis of the pancreatic cancer cells. The xenograft nude mice model bearing human pancreatic cancer Panc28 cells was used to determine the antitumor effect of exomiR-34a in vivo.

Results: The exomiR-34a could cross the cell membrane efficiently, and downregulated the expression of the targeted gene Bcl-2. Treatment with exomiR-34a inhibited the growth of the pancreatic cancer cells significantly and the nanoparticles also induced apoptosis in cancer cells via affecting the expression of apoptotic-related genes. In vivo study using xenograft nude mice bearing Panc28 cancer cells revealed that exomiR-34a suppressed the growth of tumors significantly.

Conclusion: ExomiR-34a can inhibit the growth of pancreatic cancer both in vitro and in vivo. Targeting miR-34a is a promising strategy for the treatment of pancreatic cancer. ExomiR-34a has the potential to be developed as a novel anticancer agent for the treatment of human pancreatic malignancy.

Keywords: pancreatic cancer, miR-34a, exosomes, Bcl-2, anticancer

\section{Introduction}

Pancreatic cancer, the fourth-leading cause of cancer-related deaths worldwide, is characterized by a poor prognosis, early recurrence, and metastasis. Traditional treatment of pancreatic cancer includes surgery, chemotherapy, radiation therapy, and palliative care. Although diagnostic and therapeutic measures have improved, the survival rate still remains dismal, with an overall five-year survival rate of $8 \%$ in America. ${ }^{1-3}$ Gemcitabine and 5-fluorouracil (5-Fu) have been used as the firstline chemotherapeutic agents for the treatment of pancreatic cancers in clinical settings. However, their efficacy is limited due to the drug resistance of the cancer 
cells. ${ }^{4}$ Therefore, there is an urgent need to develop a novel strategy to improve the therapeutic outcome and prolong the survival of pancreatic cancer patients.

It has been well established that the aberrant expression of miRNAs is highly related to the development of the malignancy of pancreatic cancer. ${ }^{5}$ Many studies have shown that miR-34a can act as an antioncogene; decreased miR-34a levels are associated with stronger cancer activity in pancreatic cancer cell lines, and overexpression of miR34a induced pancreatic cancer cell apoptosis and inhibited cell growth. ${ }^{6-8}$ Transfection of miR-34a mimics or infection with lentiviral miR-34-MIF in pancreatic cancer were able to reduce the invasion and metastasis of pancreatic cancer through direct modulation of the downstream targets Bcl-2 and Notch. ${ }^{6}$ In addition, miR-34a can also inhibit pancreatic cancer progression by posttranscriptionally regulating Snail1 and Notch1 expression. ${ }^{7}$ This evidence reveals that miR-34a has the potential to be developed as an anticancer agent for the treatment of human pancreatic cancer. However, poor cellular delivery of miRNAs limits their application, because of their poor membrane penetration potential, short halflife in circulation, and weak endosomal release., ${ }^{9,10}$ Therefore, developing effective miRNA delivery approaches are promising for successful gene therapy, and finding carrier systems with high efficiency and low toxicity are urgently needed in developing miRNA drugs.

Exosomes, nanosized particles with a diameter of 30-150 nm have been found in many body fluids, including blood, cerebrospinal fluid, saliva, urine, and milk. ${ }^{11}$ A previous study has shown that exosomes function as promising carriers for delivering miRNA into cells. Compared with other traditional gene carriers, exosomes possess several advantages; the phospholipid structure of exosomes is similar to the composition of the cell membrane, making it easier to cross into cells. ${ }^{12}$ Additionally, as an endogenous vesicle, exosomes usually do not result in immune response when entering the circulation of the human body. ${ }^{13}$ In the past decade, there are numerous reports to show that exosomes-mediated gene delivery is able to effectively transfer siRNA, DNA, and proteins into targeted cells. ${ }^{14}$ Treatment of zebrafish with exosomescoated-VEGF siRNA leads to an inhibitory effect of angiogenesis, and the nanoparticles also successfully cross the blood-brain barrier (BBB) in zebrafish. ${ }^{15}$ Exosomes-coated miR-128-3p is capable of downregulating of MRP5 and Bmil, re-sensitizing colorectal cancer cells to oxaliplatin, while exosomes-coated-miR-143 is able to suppress the proliferation and migration of osteosarcoma cells. ${ }^{16,17}$ Ohno et al designed modified exosomes expressing GE11, an epidermal growth factor receptor (EGFR)-binding peptide, and the modified exosome-coated let-7a was able to target breast cancer with high expression of EGFR, leading to the inhibitory effect of breast cancer. ${ }^{18}$ However, exosomes-mediated delivery of miRNAs is still in its infancy and further studies should be performed to validate the efficiency and credibility of the novel approach.

In this study, the exosomes were isolated from the conditioned medium of HEK293 cells and the exosomescoated miR-34a (exomiR-34a) was synthesized through an ultrasonic approach. The anticancer effect of exomiR-34a was studied both in vitro and in vivo, and the results showed that exomiR-34a was able to inhibit the growth of pancreatic cancer significantly.

\section{Materials and Methods Cell Culture}

Human embryonic kidney (HEK293) cells, normal human pancreatic epithelial ductal cells (HPDE6-C7), and human pancreatic cancer Miapaca-2 and Panc28 cell lines were obtained from the American Type Culture Collection (ATCC, Manassas, VA). HEK293, HPDE6-C7, and Miapaca-2 cancer cells were maintained in Dulbecco's modified Eagle's medium (DMEM) with 10\% fetal bovine serum and $1 \%$ antibiotic. Panc28 cancer cells were maintained in Roswell Park Memorial Institute (RPMI) 1640 Medium supplemented with 100 units penicillin and 100 units per $\mathrm{mL}$ streptomycin. All cells were incubated at $37^{\circ}$ $\mathrm{C}$ with $5 \% \mathrm{CO}_{2}$.

\section{Chemicals and Reagents}

Monoclonal antibodies including CD9, CD63, and TSG101 were products of Abcam (Cambridge, England). Antibodies against CD81 were obtained from Santa Cruz Biotechnology (Dallas, USA). Antibodies against GAPDH, Bcl-2, Bax, and P53 were products of Cell Signaling Technology (Danvers, MA, USA). Matrigel was purchased from BD (New Jersey, USA). Fibronectin was obtained from ACMEC Biochemical (Shanghai, China). All other chemicals used were of high purity purchased from commercial sources.

\section{Synthesis of exomiR-34a}

Exosomes were purified from the medium of HEK293 cells as described by Thery et al with little 
modification. ${ }^{19}$ Briefly, HEK293 cells were cultured in the logarithmic growth phase, and then cells were seeded in ten $75 \mathrm{~cm}^{2}$ flasks; each flask with $25 \mathrm{~mL}$ culture medium. After the cells were grown to $60-70 \%$ abundance, the original medium was removed and replaced with the same amount of fresh exosome-free serum medium. After the cell growth reached about $80-95 \%$ abundance, the supernatant was collected by centrifugation at $10,000 \times$ $\mathrm{g}$ for $30 \mathrm{~min}$ at $4^{\circ} \mathrm{C}$ to remove the residual debris, and then the supernatant was filtrated through $0.22 \mu \mathrm{M}$ polyvinylidene difluoride filters. The filtrated solution was centrifuged at $100,000 \times \mathrm{g}$ for $70 \mathrm{~min}$ at $4^{\circ} \mathrm{C}$ and the exosomes pellets thus obtained were washed twice with phosphatebuffered saline (PBS) and resuspended in $50 \mu \mathrm{L}$ PBS. Butyleyanoacrylate (BCA) protein assay kit (Thermo Scientific, CA, USA) was used to determine the total protein content of exosomes. The size distribution and zeta potential of the isolated exosomes were measured by Nano-Zetasizer 90 at $37^{\circ} \mathrm{C}$ as per the manufacturer's instructions. ${ }^{20}$ The morphology of exosomes after negative staining was observed by transmission electron microscopy. ${ }^{21}$ Western blot analysis was performed to determine the expression of the characteristic proteins of exosomes including CD81, CD63, CD9, etc. ${ }^{22}$

In order to analyze the distribution and the concentration of miRNAs, the miRNAs were labeled cy3 at the $5^{\prime}$ end of the miRNAs. The exomiR-34a was synthesized using an ultrasound approach. Briefly, the exosomes isolated above were dissolved in PBS solution, and miR-34a (mass ratio of miRNA: exosomes $=1: 5$ ) was added to the PBS solution and ultrasonicated at $20 \mathrm{~W}$, for $5 \mathrm{~s}$, stopping for $2 \mathrm{~s}$ and then the solution was allowed to stand on ice for $20 \mathrm{~min}$. The loading rate of miRNA in exosomes was measured by agarose gel electrophoresis, stained with GelRed (Vazyme, China), and analyzed by agel-imaging system (BioRad, USA).

\section{Analysis of the Distribution and Transfection Efficiency of exomiR-34a}

The distribution of exomiR-34a in cancer cells was measured using confocal microscopy. Briefly, Panc28 and Miapaca-2 cells $\left(1.5 \times 10^{5}\right)$ were seeded in a confocal dish and certain amounts of exomiR-34a were added and incubated for $24 \mathrm{~h}$. Cells were then fixed with $4 \%$ methanol-free paraformaldehyde. After incubation for $10 \mathrm{~min}$, the cells were stained with Actin-Tracker Green (Beyotime, China) and DAPI (Solarbio, China). Confocal microscopy was used to observe the distribution of the exomiR-34a.

The transfection efficiency was determined using the flow cytometry approach. Briefly, Panc28 and Miapaca-2 cells $\left(2 \times 10^{5}\right)$ were plated in six-well plates and treated without or with a certain amount of exomiR-34a for 24 h. Cells were collected by centrifugation at $1000 \times \mathrm{g}$ for 10 min and analyzed with a flow cytometer (Becton, Dickinson and Company, USA). Transfection efficiency was indicated by the proportion of cy3-positive cells.

\section{Determination of Apoptosis}

The apoptosis of cancer cells induced by exomiR-34a was analyzed by Annexin V-EGFP/PI double staining. Briefly, Panc28 and Miapaca- 2 cells $\left(2 \times 10^{5}\right)$ were seeded in sixwell plates. After being treated with a certain amount of exomiR-34a for $48 \mathrm{~h}$, cells were collected by centrifugation at $1000 \times \mathrm{g}$ for $10 \mathrm{~min}$ and stained using Annexin V-EGFP/PI double staining cell apoptosis detection kit (Keygen Technology, China) as per the manufacturer's instruction. The apoptosis rate was analyzed using a flow cytometer (Becton, Dickinson and Company, USA).

\section{Quantitative RT-PCR}

Panc28 and Miapaca-2 cells $\left(2 \times 10^{5}\right)$ were plated in sixwell plates and were treated without or with a certain amount of exomiR-34a for $24 \mathrm{~h}$. For BCl2 mRNA expression analysis, total RNA was extracted using a total RNA extraction kit (Tiangen Biotech, China) as per the manufacturer's instruction. RNA then was reverse transcribed to cDNA using a cDNA transcription kit (FastQuant RT kit, Tiangen Biotech, China). qRT-PCR analysis was performed using SuperReal Premix Pluskit (SYBR Green) (Tiangen Biotech, China) according to the manufacturer's protocol and data were analyzed by a real-time fluorescence quantitative PCR detection system IQ5 (BioRad, USA). The primers for amplification of BCL2 were as follows: BCL2 forward primer; 5'-GAC TTC TCC CGC CGC TAC CG-3', BCL2 reverse primer; 5'-ACA CAC ATG ACC CCA CCG AAC-3'. The amplification of miR-34a was performed using the miRcute Plus miRNA qPCR kit (Tiangen Biotech, China) as per the protocol. Briefly, total miRNA was extracted, and the poly A tail was added using E. coli Poly(A) Polymerase. The cDNA of miRNAs was reverse transcripted using oligo-dT universal primers. The sequence of miR-34a forward primer was as follows: 5'-UGGC AGU GUC UUA GCU GGU UGU- $3^{\prime}{ }^{23}$ The reverse poly $\mathrm{T}$ primers were involved in 
the kit. PCR amplification reaction was performed using the following conditions: denaturing at $95^{\circ} \mathrm{C}$ for $15 \mathrm{~min}$, and then $94^{\circ} \mathrm{C}$ for $20 \mathrm{~s}, 60^{\circ} \mathrm{C}$ for $30 \mathrm{~s}$, in total for 45 circles. The relative levels of miR-34a and BCL2 mRNA were normalized to the U6 snRNA/GAPDH mRNA expression, respectively. The $2^{-\Delta \Delta \mathrm{Ct}}$ method was used to analyze the real-time PCR data.

\section{Cell Proliferation Assay}

MTT assay was performed to determine the viability of pancreatic cells. Briefly, HPDE6-C7, Panc28, and Miapaca-2 cells $\left(3 \times 10^{3}\right)$ were plated in 96-well plates. After incubation for $24 \mathrm{~h}$, cells were treated with a certain amount of exomiR-34a, miR-34a, and unmodified exosomes. After being cultured for 7 days, MTT $(10 \mu \mathrm{L}$, $5 \mathrm{mg} / \mathrm{mL}$, Sigma-Aldrich, St Louis, MO) was added to each well and the cells were incubated for an additional 4 h. DMSO $(150 \mu \mathrm{L})$ was added to each well to dissolve the reduced MTT crystals. The MTT-formazan product dissolved in DMSO was estimated by measuring the absorbance at $570 \mathrm{~nm}$ with a microplate reader (Biotech, power wave, USA). The percentage of cell growth inhibition was calculated as follows: Relative inhibitory rate $(\%)=$ $\left(\mathrm{OD}_{\text {control- }}-\mathrm{OD}_{\text {treated }}\right) / \mathrm{OD}_{\text {control }} \times 100 \%$.

\section{Western Blot Analysis}

The expression of CD63, CD81, CD9, ALIX, Bcl-2, Bax, and P53 was analyzed using Western blot as described previously. ${ }^{22}$ Briefly, cells $\left(2 \times 10^{5}\right)$ were plated in six-well plates overnight. The cells were then treated without or with a certain amount of exomiR-34a. After being incubated for another $48 \mathrm{~h}$, cells were lysed with RIPA lysis buffer (Beyotime, Shanghai, China) on ice for $30 \mathrm{~min}$, and the cell lysate was centrifugated at $12,000 \mathrm{x}$ g for $15 \mathrm{~min}$ at $4{ }^{\circ} \mathrm{C}$ and the supernatant was collected. Protein concentration was measured using a BCA Protein Assay Kit (Thermo Scientific, CA, USA) and equal amounts of proteins were resolved by electrophoresis on $10 \%$ sodium dodecyl sulfate polyacrylamide gel and transferred onto polyvinylidene difluoride (PVDF) membranes (Millipore, NY, USA). The membranes were blocked with $5 \%$ non-fat milk in TBS-T (TBS, $150 \mathrm{mM} \mathrm{NaCl}, 20 \mathrm{mM}$ Tris-HCl, $\mathrm{pH}=7.4$, with $0.5 \%$ Tween 20) for $1 \mathrm{~h}$ at room temperature, subsequently incubated with primary antibodies including rabbit anti-GAPDH rabbit anti-CD63, rabbit anti-CD81, rat anti-CD9, mouse anti-ALIX, rabbit anti-Bcl-2, rabbit anti-Bax, rabbit antiP53 at $4^{\circ} \mathrm{C}$ overnight. After washing with TBS-T 3 times, the membranes were incubated with peroxidase-conjugated secondary antibodies (CWBIO, Beijing, China) for $1 \mathrm{~h}$ at room temperature. Finally, protein bands were visualized by adding the Super Signal West Dura Extended Duration Substrate (Thermo Scientific, CA, USA). The relative expression of proteins was analyzed using ImageJ software. All data were representatives of at least three independent experiments.

\section{Determination of Antitumor Activity of exomiR-34a in vivo}

All animal experiments were approved by the Institutional Animal Care and Use Committee of Southwest Medical University $(20,180,391,216)$; our animal experiments meet the standards set out in the NC3Rs primate's guidelines and follow best practice procedures. Female nude BALB/c mice (4-6 weeks, 18-20 g) were subcutaneously injected with Panc28 cells $\left(1 \times 10^{7}\right)$ at the right flanks. In total 20 mice were randomly divided into 4 groups with 5 mice in each group; the PBS control group, exosomes $(7.5 \mathrm{mg} / \mathrm{kg})$ group, exomiR-34a (1.5 mg/kg miR-34a) group, and positive 5-FU group $(5-\mathrm{Fu}, 10 \mathrm{mg} / \mathrm{kg})$. When tumors were grown up to about $80 \mathrm{~mm}^{3}$, the mice were treated i.v. with PBS, exosomes, exomiR-34a, and 5-Fu, respectively, every 4 days for 21 days. Of note, the 5-Fu was treated every other day. The tumor size and were measured every day by a Vernier caliper, and the body weight and tumor volume were analyzed using the following formula: Tumor volume $\left(\mathrm{mm}^{3}\right)=0.5 \times$ length $\times(\text { width })^{2}$, while the relative tumor growth rate $=$ tumor volume/initial tumor volume $\times 100 \%$. No mice died of tumor loading in the experiments.

\section{Immunohistochemistry and TUNEL Assay}

Mice were administrated with exosomes, exomiR-34a, lipomiR-34a, and 5-Fu as described above. After being treated for 21 days, all of the mice were sacrificed and tumors were removed. Paraformaldehyde (4\%) was used to fix the excised tumor tissues. After embedding in paraffin, the tumor tissues were cut into 4-8 $\mu \mathrm{m}$ and then stained with IHC. Anti-Ki-67 and anti-Bcl-2 antibodies were added and incubated at $4^{\circ} \mathrm{C}$ for $24 \mathrm{~h}$. Then, the biotin-labeled secondary antibody was added to the tissue sections and incubated for $1 \mathrm{~h}$. The tissue sections were stained with 3,3'diaminobenzidine (DAB) substrate. After counterstaining with hematoxylin, the tissue sections were dehydrated and sealed with coverslips and the relative expression of Ki-67 and Bcl2 was analyzed using a high-capacity digital slide scanner system (3DHISTECH Ltd., Budapest, Hungary). 
For TUNEL assay, terminal deoxynucleotidyl transferasemediated deoxyuridine triphosphate nick end labeling (TUNEL) was used to stain the paraffin tumor sections. After being dewaxed and hydrated, a proteinase $\mathrm{K}$ solution was added to the tissue sections and incubated for 15-20 min at $37^{\circ} \mathrm{C}$, and then treated with $100 \mu \mathrm{L}$ DNaseI reaction solution. Each tissue section was incubated with TdT enzyme reaction solution $(100 \mu \mathrm{L})$ at $37^{\circ} \mathrm{C}$ in the dark and humid environment for $1 \mathrm{~h}$. Then, Streptavidin-HRP $(100 \mu \mathrm{L})$ was added to incubate for $30 \mathrm{~min}$ at $37^{\circ} \mathrm{C}$. The tissue sections were then stained with $\mathrm{DAB}$ as described above. The high-capacity digital slide scanner system (3DHISTECH Ltd., Budapest, Hungary) was used to determine the apoptosis rate of cancer cells.

\section{Statistical Analysis}

Statistical analysis was carried out using SPSS 17.0 (SPSS Inc., Chicago, IL). The data are expressed as mean $\pm \mathrm{SD}$ and all of the experiments were performed at least three times. Student's $t$-test or two-way ANOVA analysis were used to analyze the difference among the data groups, and a significant difference was defined when $* p<0.05$, ** $p<0.01, * * * p<0.001$.

\section{Results}

\section{Synthesis of Exosomes and Synthesis of exomiR-34a}

Exosomes were isolated from the supernatant of HEK293 cells by differential centrifugation with a yield of $150 \mathrm{mg} /$ L. An ultrasonic approach was used to prepare the exomiR-34a. The size of exosomes was around $77.82 \mathrm{~nm}$ in diameter, while the size of exomiR-34a was around $85.42 \mathrm{~nm}$ in diameter as measured by Nano-Zetasizer 90 (Figure 1A and B). Western blot analysis revealed that there was high expression of exosomes specific markers, including TSG101, CD63, CD9, and CD81 (Figure 1C). There are a lot of inwardly concave cuplike structures in most exosomes as observed by transmission electron microscopy (Figure 1D). The RNA loading rate in exosomes reached the maximum value of $64.8 \pm 3.5 \%$ when the ratio of exosomes to miR-34a is 5:1 as analyzed by agarose gel electrophoresis (Figure 1E).

\section{ExomiR-34a Entered Cancer Cells Efficiently}

Cancer cells were treated with exomiR-34a and the distribution of exomiR-34a was determined using confocal laser- scanning microscopy. The results showed that exo-miR34a was widely distributed in both Panc28 (Figure 2A) and Miapaca-2 (Figure 2B) cells. Flow cytometry analysis showed that exomiR-34a was highly distributed in both Panc28 (Figure 2C) and Miapaca-2 (Figure 2D) cells; quantitative analysis revealed that the mean fluorescence intensity increased from around 800 to 2500 and 5000 in Panc28 cells transfected with lipofectamine3000-coated miR-34a (lipomiR-34a) and exomiR-34a, respectively, compared with the cells transfected with the parent miR-34a, while the mean fluorescence intensity increased from around 900 to 2510 and 4500 in Miapaca-2 cells transfected with lipomiR34a and exomiR-34a, respectively, compared with the cells transfected with the parent miR-34a (Figure 2E). The relative transfection efficiency increased to $42 \%$ and $86 \%$ in Panc 28 cells transfected with lipomiR-34a and exomiR-34a, respectively, compared with the cells transfected with the parent miR-34a. Similar results were also obtained in Miapaca-2 cells (Figure 2F). The results suggested that exomiR-34a is able to cross the cell membrane with higher efficiency.

\section{ExomiR-34a Inhibited the Growth of Cancer Cells Significantly}

We then examined the effect of exomiR-34a on the growth of pancreatic cancer cells. MTT assay showed that the transfection of exomiR-34a significantly inhibited the growth of cancer cells; the inhibitory rates were $79 \%$ and $67 \%$ in Panc28 (Figure 3A) and Miapaca-2 cells (Figure 3B), respectively, when treated with exomiR-34a for 7 days. MiR-34a and the unmodified exosomes treatment have no significant growth inhibitory effects on the growth of pancreatic cancer cells. It is very interesting that the exomiR-34a treatment displayed very weak cytotoxicity towards HPDE6-C7 cells (Figure 3C). This result indicated that exomiR-34a was able to inhibit the growth of pancreatic cancer cells with some specificity. To investigate the underlying mechanism of miR-34a on the inhibitory effects of pancreatic cancer cells, we detected the expression of miR$34 \mathrm{a}$ in the pancreatic ductal epithelial cells as well as Panc28 and Miapaca-2 cancer cells. The qRT-PCR result showed that compared with normal pancreatic HPDE6-C7 cells, low expression of miR-34a was found (Figure 3D), while high expression of $\mathrm{Bcl} 2$, the targeted gene of miR-34a, was found in both Panc28 and Miapaca-2 cells (Figure 3E). Subsequently, a qRT-PCR assay was performed to detect the level of miR-34a and BCl2 mRNA after treated with exomiR-34a. The results show the transfection of exomiR- 
A

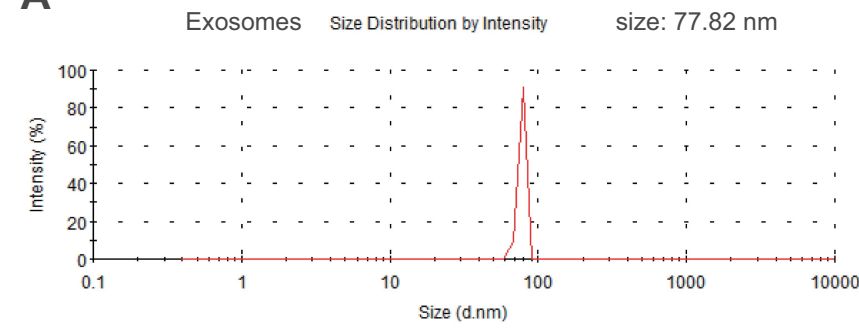

B

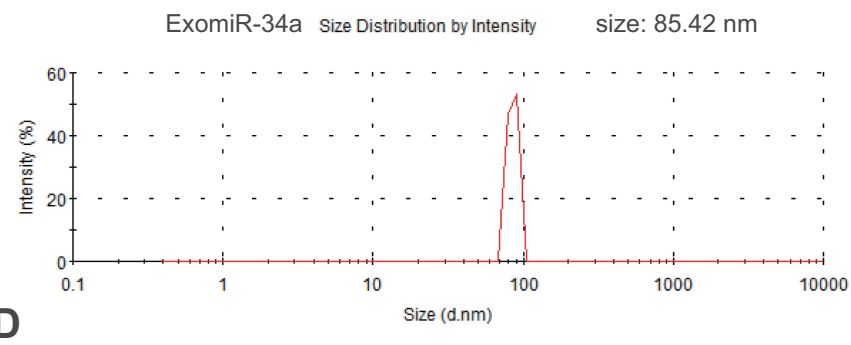

Exosomes

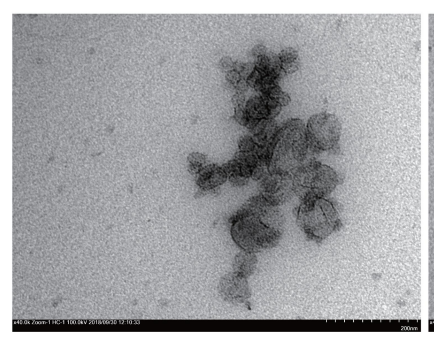

ExomiR-34a

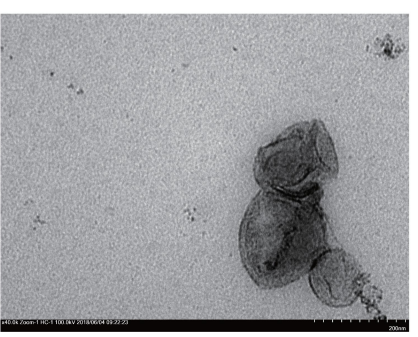

C

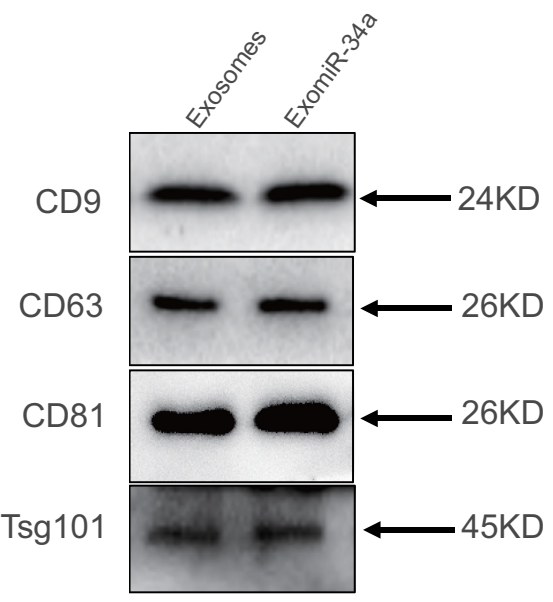

E

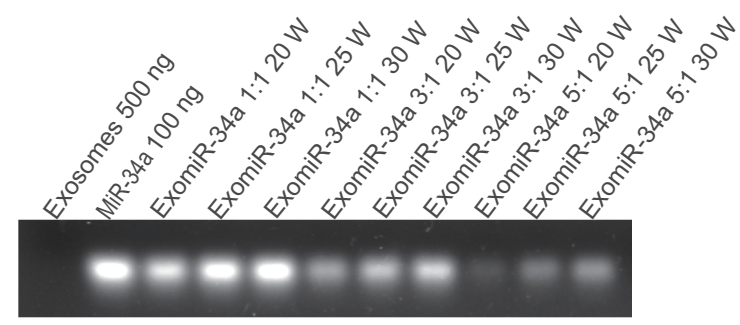

Figure I Synthesis and characterization of exomiR-34a. Exosomes were isolated from supernatant of HEK293 cells as described in Material and Methods section. An ultrasound method was used to synthesize the exomiR-34a. The properties of exosomes and exomiR-34a were analyzed by Zetasizer (A and B). The expression of specific markers of exosomes and exomiR-34a was determined by Western blot (C). The morphology of exosomes and exomiR-34a were observed under transmission electron microscopy (D). The loading rate of miR-34a in exomiR-34a was determined using agarose gel electrophoresis (E).

$34 \mathrm{a}$ increased the level of miR-34a for more than 50 times in Panc28 (Figure 3F) while the expression of its targeted gene, BCL2, was decreased more than $90 \%$ in Panc 28 cells (Figure 3H). Similar results were also found in Miapaca-2 cells transfected with exomiR-34a (Figure 3G and I). These results suggested that exomiR-34a is able to inhibit cancer cell growth via targeting BCL2.

\section{ExomiR-34a Induced Apoptosis of Cancer Cells}

In order to determine the proapoptotic effect of exomiR-34a on cancer cells, flow cytometry analysis with double staining of Annexin-V/PI of the cancer cells was carried out. As shown in Figure 4A and B, compared with control, transfection of exomiR-34a led to significant augmentation of apoptotic cell population; the apoptotic rates were $21.6 \pm 5.2 \%$ and $12.2 \pm$ 2.26\%, respectively, in Panc28 (Figure 4C) and Miapaca-2 (Figure 4D) cancer cells transfected with exomiR-34a. To further investigate the underlying mechanisms of apoptosis induced by exomiR-34a on cancer cells, Western blot analysis was carried out to check the effect of exomiR-34a on the expression of apoptotic-related genes. The results showed that the transfection of exomiR-34a resulted in the downregulation of Bcl-2 protein significantly in both Miapaca-2 and Panc28 cells (Figure 4E and F), while the expression of pro-apoptotic protein such as Bax and P53 was significantly enhanced. The results suggest that the induced apoptotic effect of exomiR$34 \mathrm{a}$ is associated with targeting apoptotic genes.

\section{ExomiR-34a Inhibited Tumor Growth in vivo}

The effect of exomiR-34a in vivo was evaluated by the xenograft nude mice model. Mice were treated with exomiR-34a i.v. and tumor volume (Figure 5A) and mouse body weight (Figure 5B) were checked every other day for 21 days. As shown in Figure 5A and C, the administration of exomiR-34a i.v. inhibited the tumor growth significantly; the inhibition rate of tumor 
A

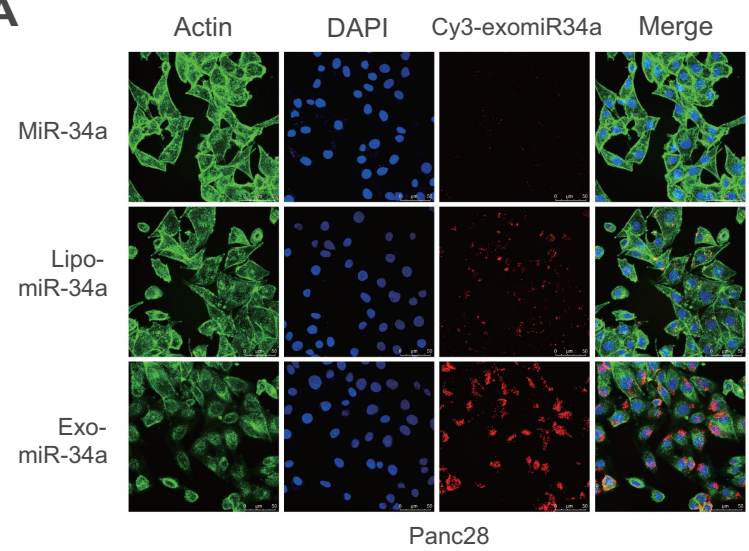

C
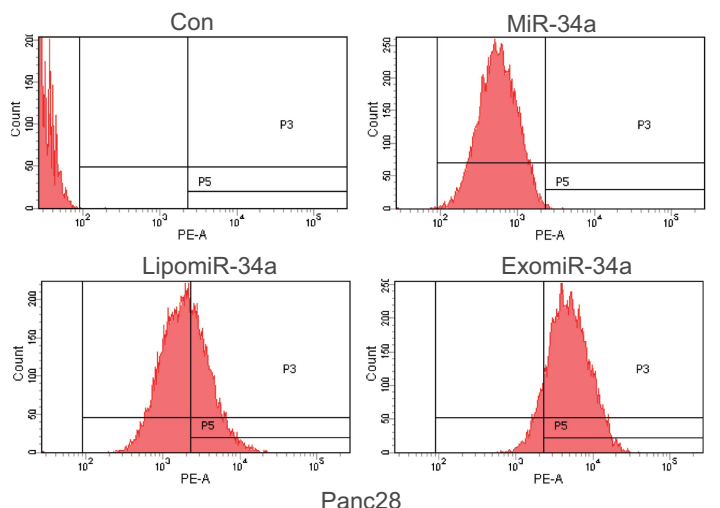

$\mathbf{E}$

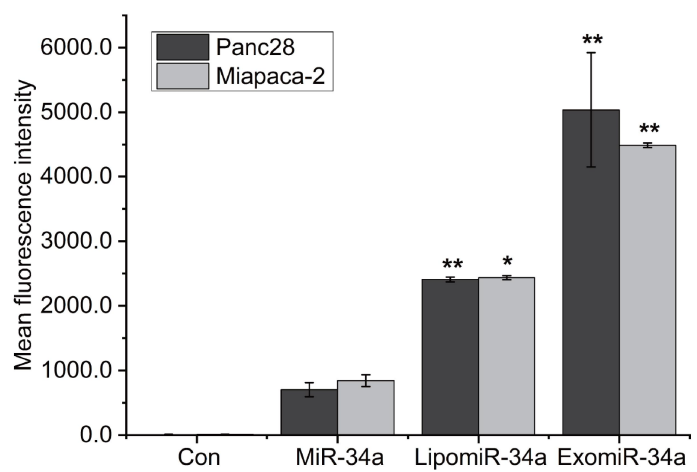

B

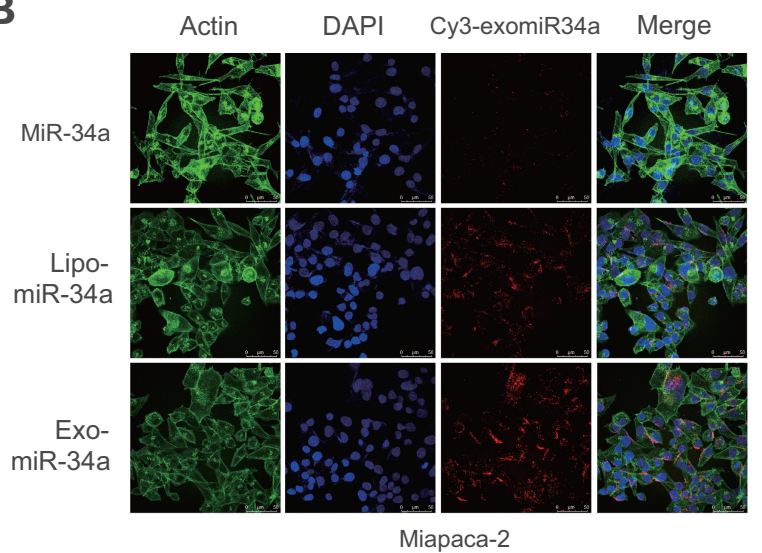

D
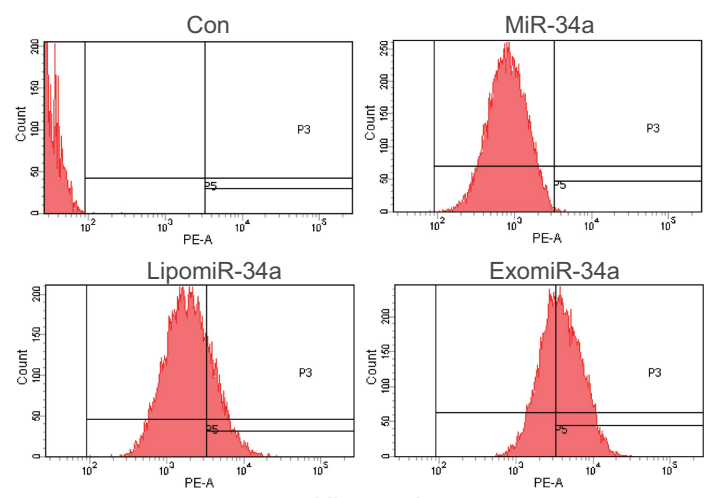

Miapaca-2

F

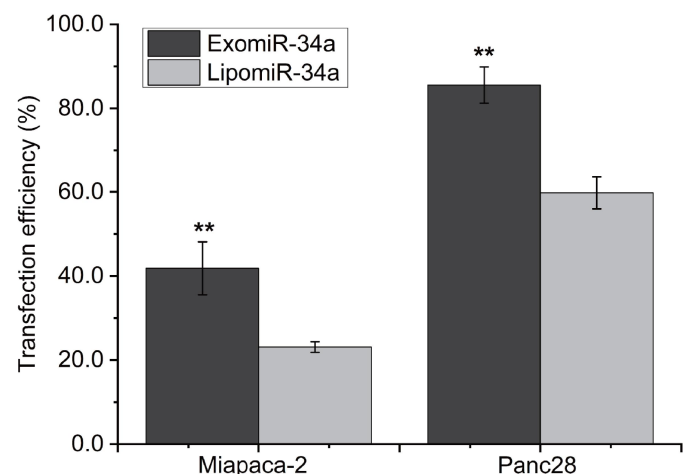

Figure 2 ExomiR-34a entered cancer cells efficiently. Pancreatic cancer cells were treated with exomiR-34a for 24 h, and the distribution of exomiR-34a in Panc28 cells (A) and Miapaca-2 (B) cells was analyzed using confocal microscopy approach. Flow cytometry analysis was carried out to determine the transfection efficiency of exomiR-34a in cells in Panc28 cells (C) and Miapaca-2 cells (D). (E) The fluorescence intensity in cells transfected with parent miR-34a, LipomiR-34a and exomiR-34a. (F) The quantitative results of transfection efficiency of $(\mathbf{C})$ and $(\mathbf{D})$. Mean $\pm S D, n=3, * P<0.05, * * p<0.01$.

growth was $86 \%$ in mice treated with exomiR-34a at day 21, a little better than that of the $5-\mathrm{Fu}$ group (Figure 5D). Of note, treatment of the mice with exomiR-34a did not affect the bodyweight of mice (Figure 5B); suggesting the low toxicity of the exosomes-coated miRNA on treated mice.

Immunohistochemical staining experiments were also performed to examine the expressions of Ki67 and $\mathrm{Bcl}-2$ in tumor tissue. The results showed that the levels of Ki67 and Bcl-2 were significantly reduced in mice treated with the exomiR-34a, compared with the control groups (Figure 6); the number of Ki67positive cells was decreased from $72.43 \pm 12.83 \%$ in the control group to $20.27 \pm 0.90 \%$ in tumor tissue treated with $1.5 \mathrm{mg} / \mathrm{kg}$ exomiR-34a. Additionally, when treated with exomiR-34a $(1.5 \mathrm{mg} / \mathrm{kg})$, the number of Bcl-2-positive cells decreased to $22.53 \pm 2.63 \%$ in tumor tissue from $85.26 \pm 7.36 \%$ in the control group. 
A

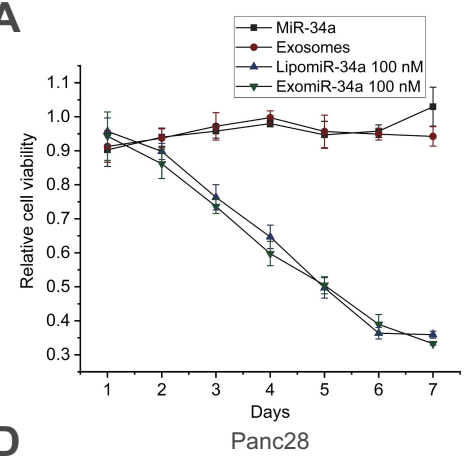

D

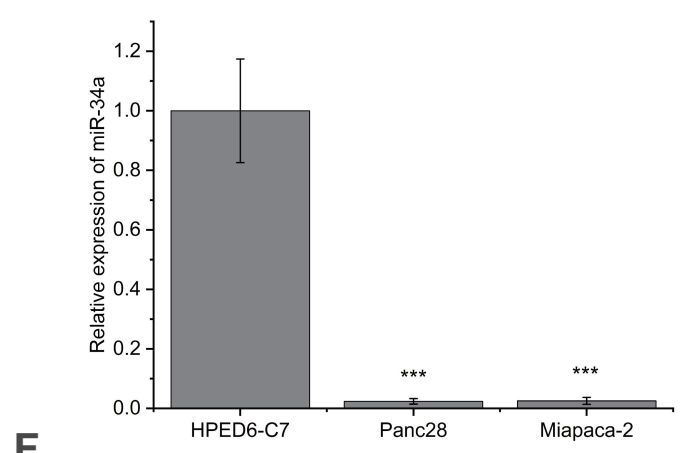

F
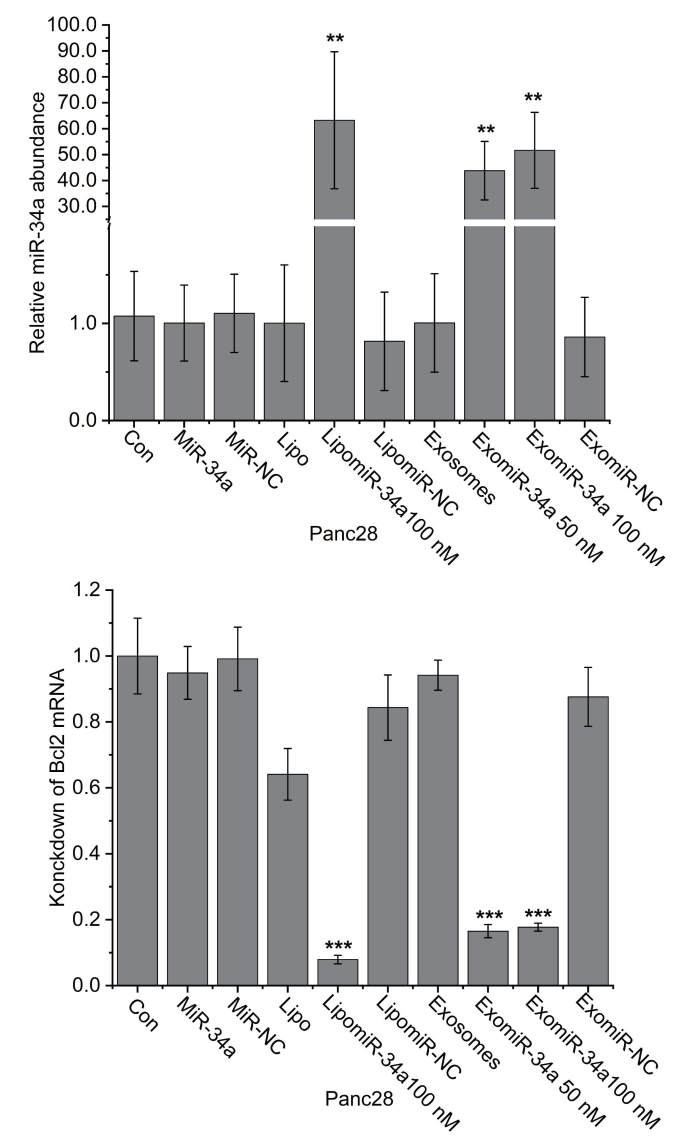

B

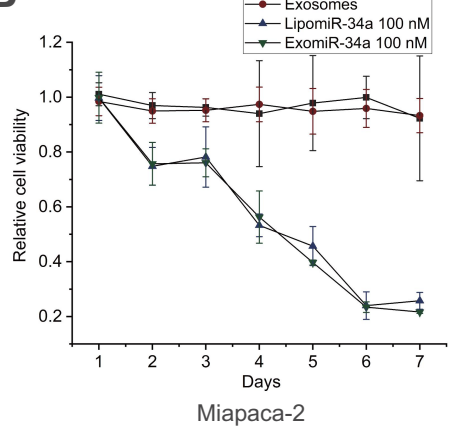

E
C

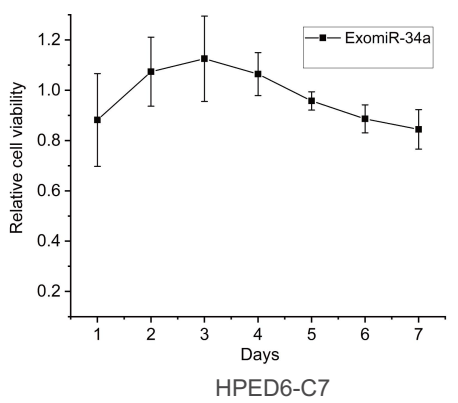

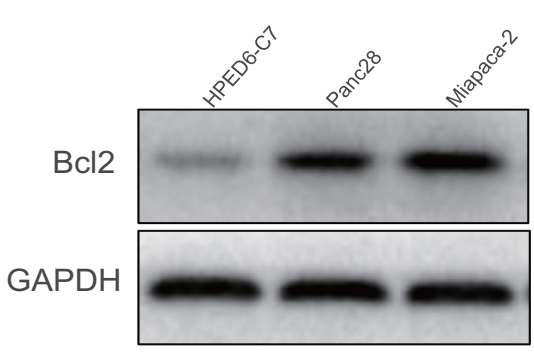

G
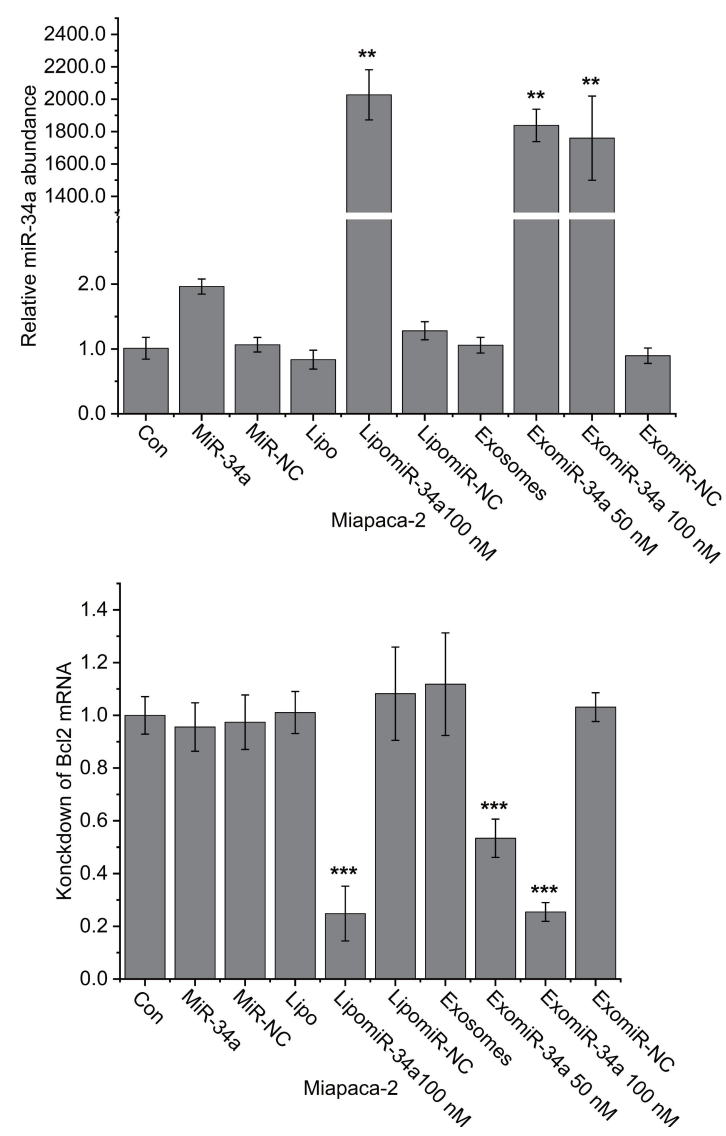

Figure 3 ExomiR-34a inhibited the growth of cancer cells significantly. Human pancreatic cancer cell lines Panc28 (A), Miapaca-2 (B) and normal human pancreatic epithelial ductal cell HPDE6-C7 (C) were treated with exomiR-34a, miR-34a, and unmodified exosomes treatment as the negative control in pancreatic cancer cells, and the cell viability was analyzed using the MTT approach. qRT-PCR analysis was carried out to determine the expression of miR-34a in HPDE6-C7, Panc28, and Miapaca-2 (D). Western blot analysis was performed to determine the initial expression levels of Bcl-2 in Panc28 and Miapaca-2 as well as HPDE6-C7 cells (E). qRT-PCR analysis was performed to determine the levels of miR-34a in Panc28 (F) and Miapaca-2 cells $(\mathbf{G})$. BCl2 mRNA expression in Panc28 (H) and Miapaca-2 cells (I) was also determined using qRT-PCR approach $(n=4), * * P<0.01$, ***P $<0.001$. 
A
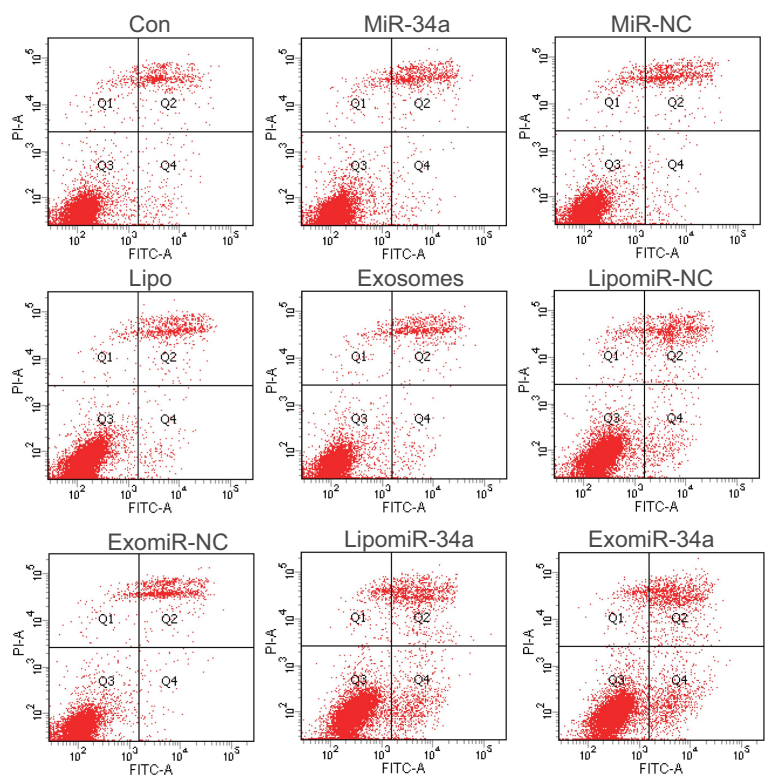

C

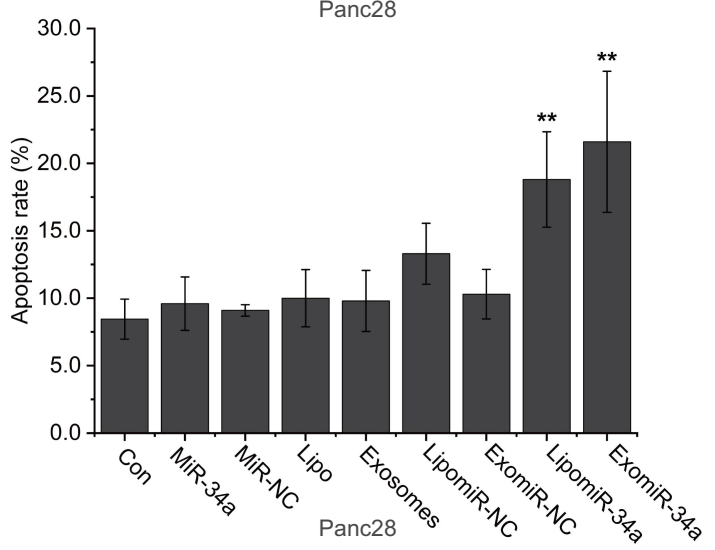

E

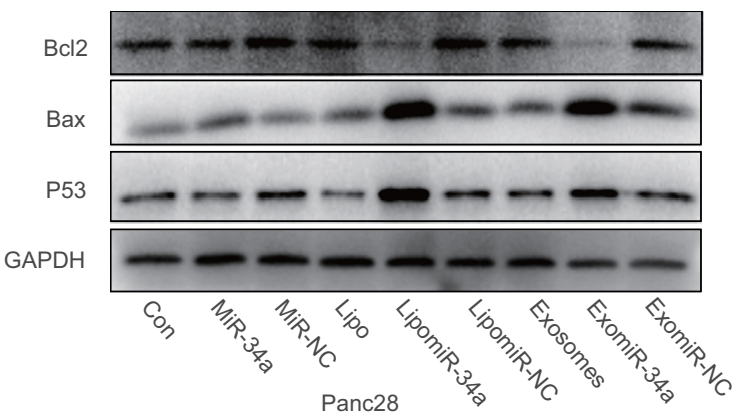

B
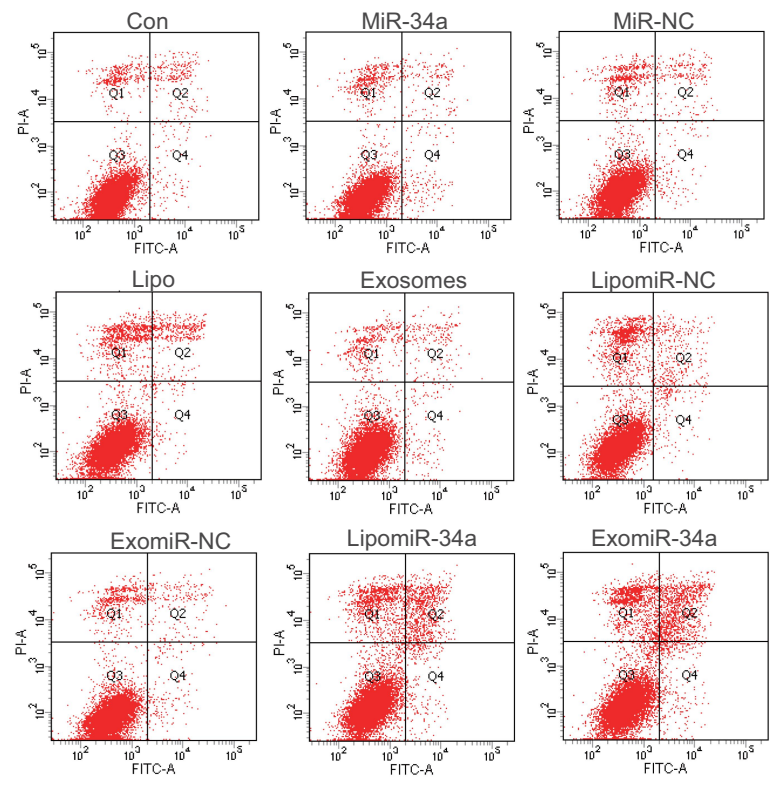

D

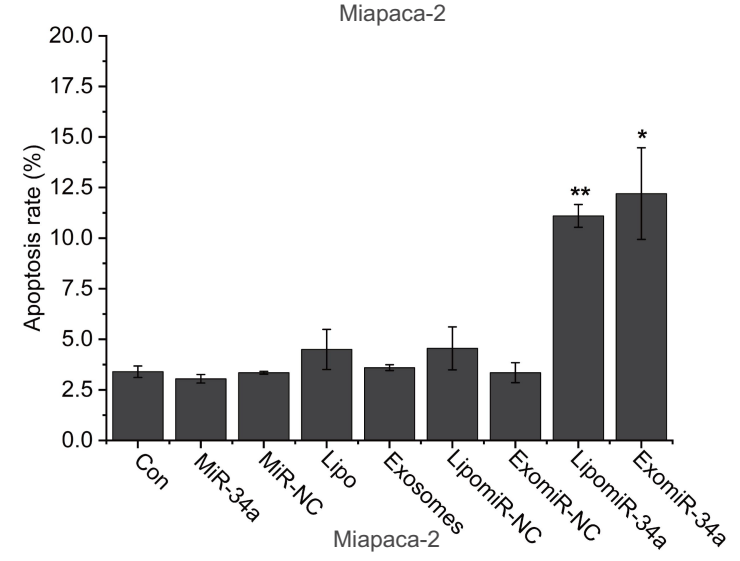

F

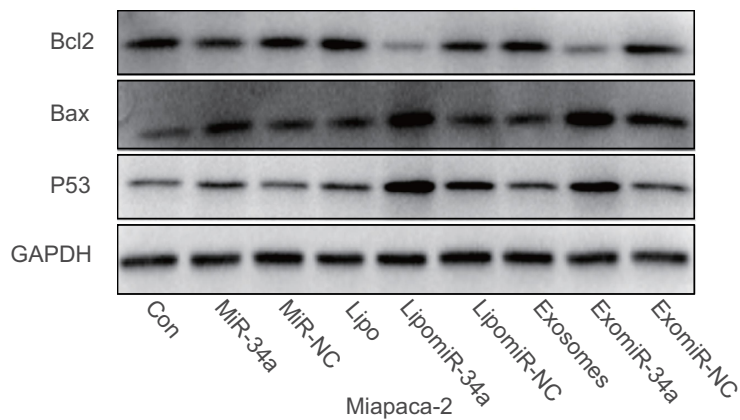

Figure 4 ExomiR-34a induced apoptosis of cancer cells. Pancreatic cancer cells were treated with exomiR-34a and flow cytometry analysis was carried out to determine the apoptosis of Panc28 (A) and Miapaca-2 (B) cells, respectively. The quantitative analysis of apoptotic rates of Panc28 (C) and Miapaca-2 (D) cells was performed. Western blot analysis was performed to determine the expression of some apoptosis-related proteins in Panc28 (E) and Miapaca-2 (F) cells. More than three independent experiments were performed for each of the research topic. Mean $\pm S D, * P<0.05$, $* * p<0.01$.

Moreover, treatment of the mice with exomiR-34a increased the apoptosis of tumor tissues significantly as determined using TUNEL assay; the apoptosis rate of the exomiR-34a-treated group increased to $59.74 \pm$ $5.15 \%$, compared with $11.23 \pm 2.26 \%$ in the control group (Figure 6). These results revealed that exomiR34a treatment was able to inhibit tumor growth significantly via inducing apoptosis mediated by downregulation of Bcl-2 expression in xenograft nude mice bearing Panc28 cells. 
A

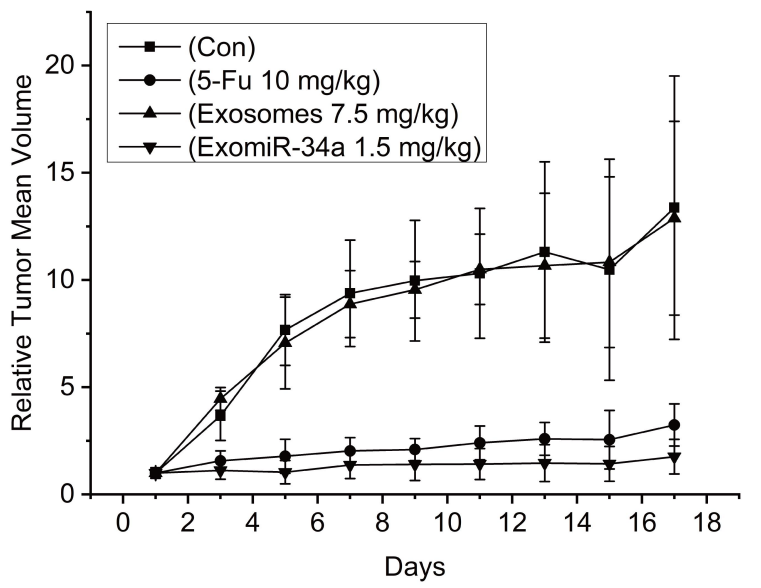

C

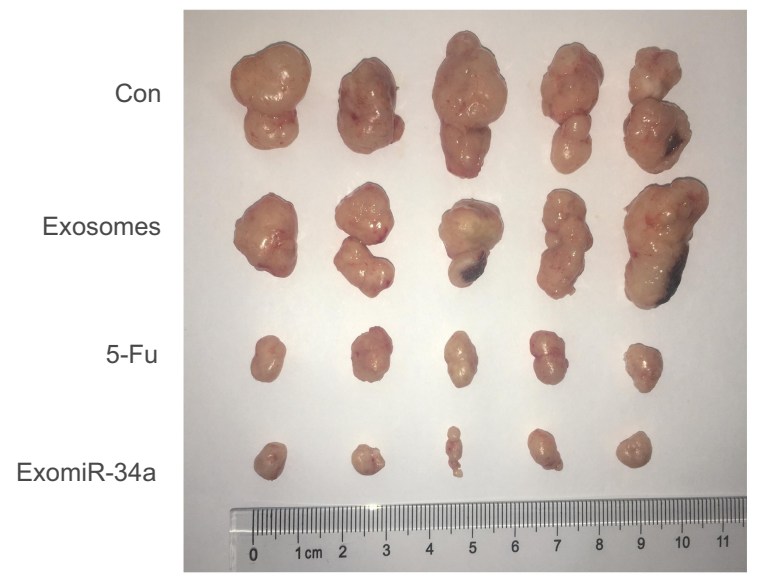

B

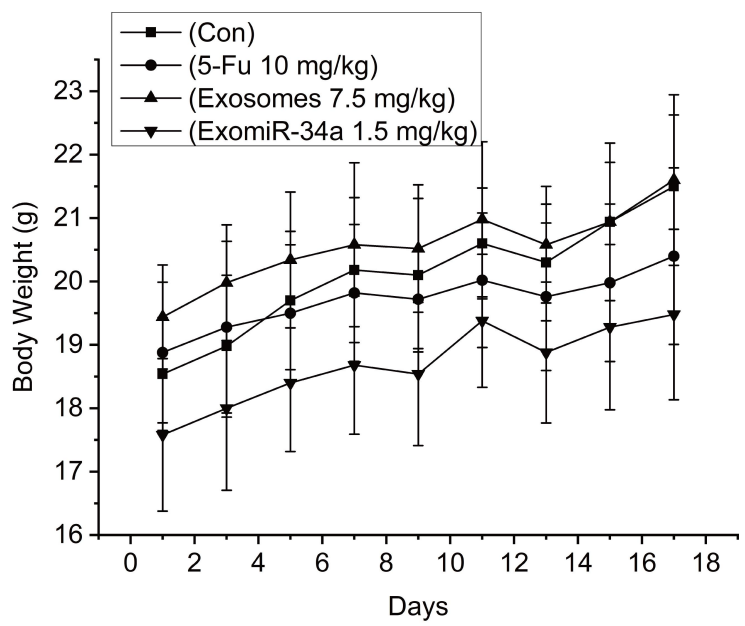

D

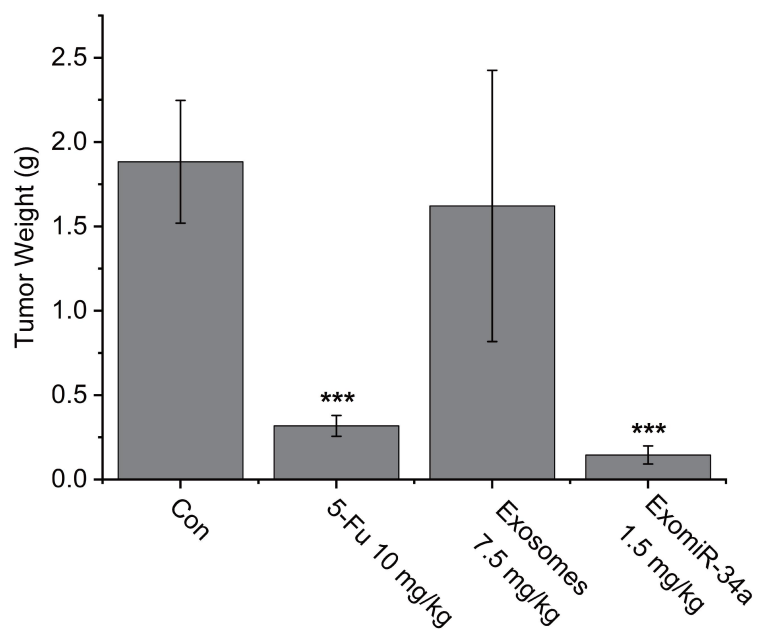

Figure 5 ExomiR-34a inhibited tumor growth of xenograft nude mice model. The Xenograft nude mice model bearing Panc28 cells was established and the model mice were injected iv with certain amount of exomiR-34a. Tumor volume (A) and mouse body weight (B) were measured every other day. After being treated with exomiR-34a for $2 \mathrm{I}$ days, the tumor tissues were removed and photographed (C). (D) The quantitative analysis of tumor weight after treating the mice with exomiR-34a for 21 days. Mean $\pm S D, n=5, * * * P<0.001$.

\section{Discussion}

It has been well documented that miRNAs play an important role in cell development, mitosis, angiogenesis, apoptosis, and metastasis. Some miRNAs acting as antioncogenes have the potential to be developed as anticancer agents. ${ }^{9}$ However, due to the large polarity of miRNA molecules, it is difficult for miRNA to enter cells, so developing effective vectors is helpful to develop miRNA drugs.

Over the past decades, a variety of vectors have been developed, including polyethyleneimine (PEI) and polyethylene glycol (PEG) mediated nanoparticles. In our previous study, we developed PEI-coated miRNA to interfere with the RNA expression, and the results showed that PEI- coated miR-125b antisense can significantly inhibit cell growth. ${ }^{24}$ However, since PEI shows strong toxicity to human normal cells, the use of PEI as a miRNA carrier cannot meet the requirement of clinical application. ${ }^{25}$ Exosomes have been developed as vehicles for the successful delivery of miRNA into cells. Due to the small size, exosomes can avoid the phagocytosis of macrophages. Compared with polymeric nanoparticles and liposomes, they are naturally stable and can escape endosomal-lysosomal degradation. In general, due to the lack of immunogenicity, these features make exosomes promising candidates for gene drug delivery. In addition, many proteins on the surface of exosomes can be modified 

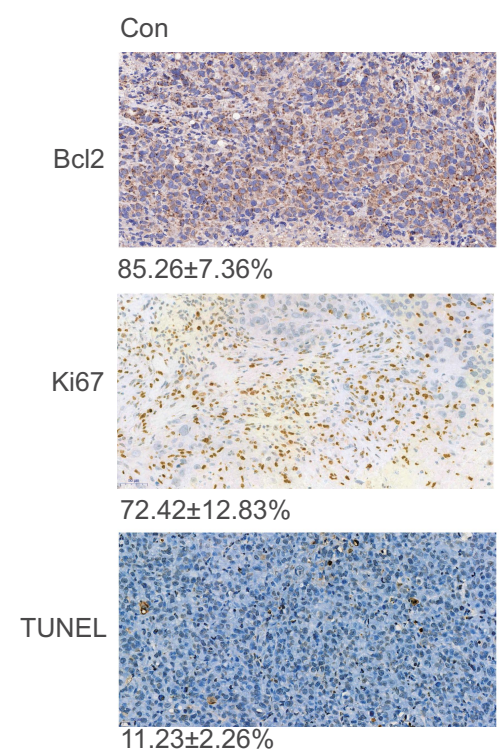

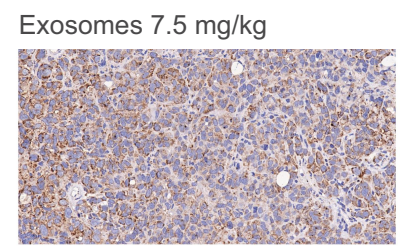

$82.66 \pm 5.37 \%$

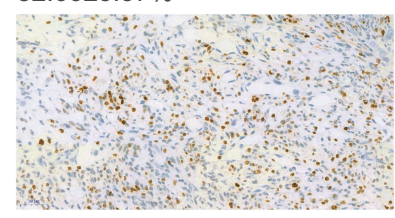

$71.29 \pm 10.24 \%$

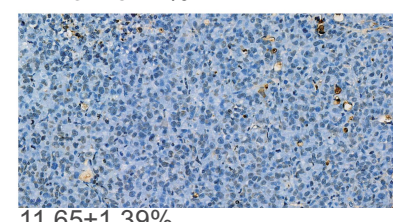

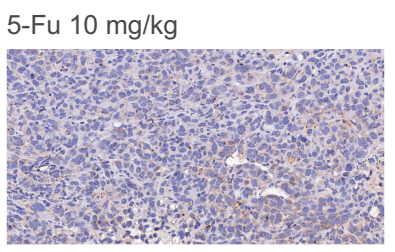

$32.27 \pm 6.57 \%$

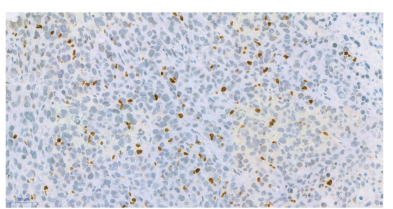

$21.06 \pm 0.59 \%$

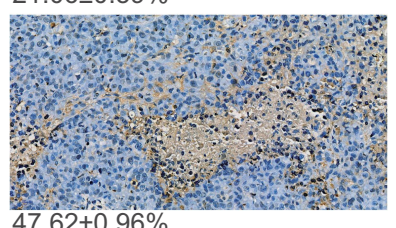

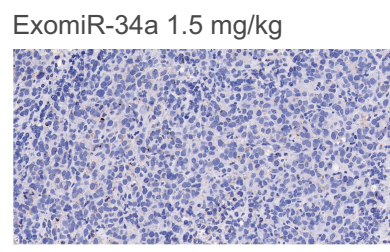

$22.53 \pm 2.63 \%$

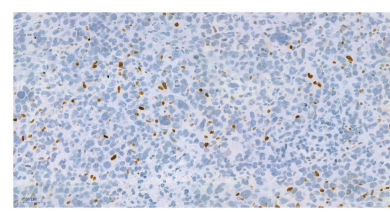

$20.27 \pm 0.90 \%$

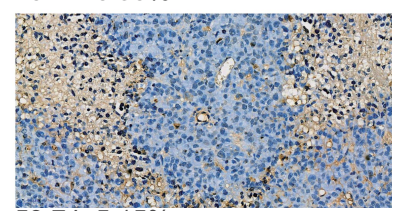

$59.74 \pm 5.15 \%$

Figure 6 Immunohistochemical (IHC) and TUNEL assay of tumors in vivo. Tumor tissues excised from Panc28 tumor-bearing mice were analyzed using IHC analysis and TUNEL assay as described in the Materials and Methods section. The level of Ki67 and $\mathrm{Bcl}-2$ protein expression in the tumor tissues was determined using $\mathrm{IHC}$ staining approach. The apoptosis rate of tumor tissues was detected using a TUNEL assay. Data are shown as mean \pm SD. $n \geq 3$.

to achieve targeted therapy in vivo. A fusion protein, lysosomal-associated membrane glycoprotein $2 \mathrm{~b}$ (Lamp2b)-rabies virus glycoprotein (RVG) was expressed, and the modified exosomes-coated miR-124 facilitated the acquisition of neuronal characteristics of cortical neural progenitor cells and prevented ischemic injury through robust cortical neurogenesis. ${ }^{26}$ The GE11 peptide modified exosome-coated let-7a was able to target breast cancer with high expression of EGFR, and inhibited the growth of breast cancer significantly. ${ }^{18}$ Studies are ongoing in our laboratory to develop modified exosomes with targeted therapy.

MiR-34a is located in the region of chromosome $1 \mathrm{p} 36.23$, which is commonly deleted in many tumor types. Ectopic miR-34a expression induces apoptosis, cell cycle arrest, and differentiation, or reduces migration. ${ }^{27}$ MiR-34a regulates a plethora of target proteins, which are involved in the cell cycle, apoptosis, differentiation, and cellular development. Besides targeting Bcl-2, miR-34a also downregulates the expression of YY1 and Notch1/2. YY1, a ubiquitous transcription factor, is negatively regulated by $\mathrm{p} 53 .{ }^{28} \mathrm{MiR}-$ $34 \mathrm{a}$ is able to bind to the $3^{\prime}$-UTR of Notch $1 / 2$, downregulating the expression of Notch1 and Notch2 proteins. It has been accepted that epithelial-mesenchymal transition (EMT) and Notch signaling play an important role in the growth and invasion of pancreatic cancer. A recent study showed that miR-34a can inhibit pancreatic cancer progression through Snaill-mediated epithelial-mesenchymal transition and the
Notch signaling pathway. ${ }^{29}$ MiR-34a inhibited the migration and invasion of pancreatic cancer cell lines while overexpression of Snail could reverse the effect of miR-34a in pancreatic cancer cells. ${ }^{7}$ More studies are needed to address if the function of exomiR-34a on pancreatic cancer is associated with the expression of YY1, Notch1/2, and Snail.

Pancreatic cancer is the fourth-leading cause of cancerrelated death in the United States. ${ }^{1}$ Up till today, there are no effective approaches to improve the outcome for the treatment of human pancreatic cancer. It is established that the aberrant expression of miRNAs is an important property of pancreatic cancer; more than 20 differential expressions of miRNAs have been found in pancreatic cancer tissues, including miR-100, miR-155, miR345, and let-7d, etc., ${ }^{30}$ suggesting that miRNAs-based therapy may be a promising approach for the treatment of pancreatic malignancy. Our result confirmed that exomiR-34a was able to inhibit tumor growth of pancreatic cancer significantly both in vitro and in vivo. The study provides a novel strategy for the treatment of human pancreatic cancer.

\section{Abbreviations}

5-Fu, 5-fluorouracil; HEK293, human embryonic kidney293; Exo, exosomes; Lipo, lipofectamine; LipomiR-NC, lipofectamine-coated miR-NC; ExomiR-NC, exosomes-coated miR-NC; LipomiR-34a, lipofectamine3000-coated miR34a; ExomiR-34a, exosomes-coated miR-34a; qRT-PCR, quantitative real-time PCR. 


\section{Acknowledgments}

The work is also supported by the Taishan Talents project of Shandong province and the Department of Science and Technology in Shandong Province of China (No\#: ZR2017MH117 and 2018YYSP025). We are also grateful for the support by the Department of Science and Technology in Sichuan of China (No\#: 2017HH0104, and 2019YFS0116) and the International Collaborative Project of the MOST of China (No\#: 2017YFE0195000).

\section{Disclosure}

The authors report no conflicts of interest in this work.

\section{References}

1. Bray F, Ferlay J, Soerjomataram I, Siegel RL, Torre LA, Jemal A. Global cancer statistics 2018: GLOBOCAN estimates of incidence and mortality worldwide for 36 cancers in 185 countries. $C A$ Cancer J Clin. 2018;68(6):394-424. doi:10.3322/caac.21492

2. Golan T, Hammel P, Reni M, et al. Maintenance olaparib for germline BRCA-mutated metastatic pancreatic cancer. $N$ Engl J Med. 2019;381(4):317-327. doi:10.1056/NEJMoa1903387

3. Ilic M, Ilic I. Epidemiology of pancreatic cancer. World J Gastroenterol. 2016;22(44):9694-9705. doi:10.3748/wjg.v22.i44.9694

4. Hong JC, Czito BG, Willett CG, Palta M. A current perspective on stereotactic body radiation therapy for pancreatic cancer. Onco Targets Ther. 2016;9:6733-6739. doi:10.2147/OTT.S99826

5. Gong R, Jiang Y. Non-coding RNAs in pancreatic ductal adenocarcinoma. Front Oncol. 2020;10:309. doi:10.3389/fonc.2020.00309

6. Ji Q, Hao X, Zhang M, et al. MicroRNA miR-34 inhibits human pancreatic cancer tumor-initiating cells. PLoS One. 2009;4(8):e6816. doi:10.1371/journal.pone.0006816

7. Tang Y, Tang Y, Cheng Y-S. miR-34a inhibits pancreatic cancer progression through Snail1-mediated epithelial-mesenchymal transition and the notch signaling pathway. Sci Rep. 2017;7(1):38232. doi:10.1038/srep38232

8. Alemar B, Izetti P, Gregorio C, et al. miRNA-21 and miRNA-34a are potential minimally invasive biomarkers for the diagnosis of pancreatic ductal adenocarcinoma. Pancreas. 2016;45(1):84-92. doi:10.1097/MPA.0000000000000383

9. Bartel DP. MicroRNAs: genomics, biogenesis, mechanism, and function. Cell. 2004;116(2):281-297. doi:10.1016/S0092-8674(04)00045-5

10. Ambros V. The functions of animal microRNAs. Nature. 2004;431 (7006):350-355. doi:10.1038/nature02871

11. Jeppesen DK, Fenix AM, Franklin JL, et al. Reassessment of exosome composition. Cell. 2019;177(2):428-445 e418. doi:10.1016/j. cell.2019.02.029

12. Van der Meel R, Fens MH, Vader P, van Solinge WW, EniolaAdefeso O, Schiffelers RM. Extracellular vesicles as drug delivery systems: lessons from the liposome field. $J$ Controlled Release. 2014;195:72-85. doi:10.1016/j.jconrel.2014.07.049

13. Valadi H, Ekstrom K, Bossios A, Sjostrand M, Lee JJ, Lotvall JO. Exosome-mediated transfer of mRNAs and microRNAs is a novel mechanism of genetic exchange between cells. Nat Cell Biol. 2007;9 (6):654-659. doi:10.1038/ncb1596
14. Jiang XC, Gao JQ. Exosomes as novel bio-carriers for gene and drug delivery. Int J Pharm. 2017;521(1):167-175. doi:10.1016/j. ijpharm.2017.02.038

15. Alvarez-Erviti L, Seow Y, Yin H, Betts C, Lakhal S, Wood MJ. Delivery of siRNA to the mouse brain by systemic injection of targeted exosomes. Nat Biotechnol. 2011;29(4):341-345. doi: $10.1038 /$ nbt. 1807

16. Shimbo K, Miyaki S, Ishitobi H, et al. Exosome-formed synthetic microRNA-143 is transferred to osteosarcoma cells and inhibits their migration. Biochem Biophys Res Commun. 2014;445(2):381-387. doi:10.1016/j.bbrc.2014.02.007

17. Liu T, Zhang X, Du L, et al. Exosome-transmitted miR-128-3p increase chemosensitivity of oxaliplatin-resistant colorectal cancer. Mol Cancer. 2019;18(1):43. doi:10.1186/s12943-019-0981-7

18. Ohno S, Takanashi M, Sudo K, et al. Systemically injected exosomes targeted to EGFR deliver antitumor microRNA to breast cancer cells. Mol Ther. 2013;21(1):185-191. doi:10.1038/mt.2012.180

19. Thery C, Amigorena S, Raposo G, Clayton A. Isolation and characterization of exosomes from cell culture supernatants and biological fluids. Curr Protoc Cell Biol. 2006; Chapter 3:Unit 322.

20. Sokolova V, Ludwig AK, Hornung S, et al. Characterisation of exosomes derived from human cells by nanoparticle tracking analysis and scanning electron microscopy. Colloids Surf B Biointerfaces. 2011;87(1):146-150. doi:10.1016/j.colsurfb.2011.05.013

21. Jung MK, Mun JY. Sample preparation and imaging of exosomes by transmission electron microscopy. J Vis Exp. 2018;131:e56482.

22. Witwer KW, Buzas EI, Bemis LT, et al. Standardization of sample collection, isolation and analysis methods in extracellular vesicle research. J Extracell Vesicles. 2013;2(1):20360. doi:10.3402/jev.v2i0.20360

23. Hermeking H. The miR-34 family in cancer and apoptosis. Cell Death Differ. 2010;17(2):193-199. doi:10.1038/cdd.2009.56

24. Rytblat I, Wu N, Xu HL, Gedanken A, Lin XK. In vitro studies of polyethyleneimine coated miRNA microspheres as anticancer agents. Nano Res. 2016;9(6):1609-1617. doi:10.1007/s12274-016-1055-4

25. Xiong MP, Forrest ML, Ton G, Zhao A, Davies NM, Kwon GS. Poly (aspartate-g-PEI800), a polyethylenimine analogue of low toxicity and high transfection efficiency for gene delivery. Biomaterials. 2007;28(32):4889-4900. doi:10.1016/j.biomaterials.2007.07.043

26. Yang J, Zhang X, Chen X, Wang L, Yang G. Exosome mediated delivery of miR-124 promotes neurogenesis after ischemia. Mol Ther Nucleic Acids. 2017;7:278-287. doi:10.1016/j.omtn.2017.04.010

27. Jamieson NB, Morran DC, Morton JP, et al. MicroRNA molecular profiles associated with diagnosis, clinicopathologic criteria, and overall survival in patients with resectable pancreatic ductal adenocarcinoma. Clin Cancer Drugs. 2012;18(2):534-545. doi:10.1158/1078-0432.CCR-11-0679

28. Chen F, Hu SJ. Effect of microRNA-34a in cell cycle, differentiation, and apoptosis: a review. J Biochem Mol Toxicol. 2012;26(2):79-86. doi:10.1002/jbt.20412

29. Rokavec M, Li H, Jiang L, Hermeking H. The p53/miR-34 axis in development and disease. J Mol Cell Biol. 2014;6(3):214-230. doi: $10.1093 / \mathrm{jmcb} / \mathrm{mju} 003$

30. Lee EJ, Gusev Y, Jiang J, et al. Expression profiling identifies microRNA signature in pancreatic cancer. Int $J$ Cancer. 2007;120 (5):1046-1054. doi:10.1002/ijc.22394 


\section{Publish your work in this journal}

Drug Design, Development and Therapy is an international, peerreviewed open-access journal that spans the spectrum of drug design and development through to clinical applications. Clinical outcomes, patient safety, and programs for the development and effective, safe, and sustained use of medicines are a feature of the journal, which has also been accepted for indexing on PubMed Central. The manuscript management system is completely online and includes a very quick and fair peer-review system, which is all easy to use. Visit http://www. dovepress.com/testimonials.php to read real quotes from published authors. 